\title{
La formule « l'armée, le peuple et la Résistance »
}

Un coup de force linguistique et politique

The formula "the army, the people and the Resistance": A linguistic and political coup de force

Pascale Asmar

\section{(2) OpenEdition}

Journals

Édition électronique

URL : http://journals.openedition.org/tipa/1947

DOI : 10.4000/tipa.1947

ISSN : 2264-7082

Éditeur

Laboratoire Parole et Langage

Référence électronique

Pascale Asmar, «La formule «l'armée, le peuple et la Résistance» », TIPA. Travaux interdisciplinaires sur la parole et le langage [En ligne], 33 | 2017, mis en ligne le 16 octobre 2017, consulté le 26 septembre 2020. URL : http://journals.openedition.org/tipa/1947 ; DOI : https://doi.org/10.4000/tipa 1947

Ce document a été généré automatiquement le 26 septembre 2020.

\section{(i) $\$ 9$}

La revue TIPA. Travaux interdisciplinaires sur la parole et le langage est mise à disposition selon les termes de la licence Creative Commons Attribution - Pas d'Utilisation Commerciale - Pas de Modification 4.0 International. 


\section{La formule « l'armée, le peuple et la Résistance »}

Un coup de force linguistique et politique

The formula "the army, the people and the Resistance": A linguistic and political coup de force

Pascale Asmar

\section{NOTE DE L'AUTEUR}

Abréviations : L'Orient-Le Jour (OLJ) ; Le Figaro (LF), Le Monde (LM), The Washington Post (WP), The New York Times (NYT), An Nahar (AN), Al Akhbar (AK)

\section{Contexte d'émergence et de diffusion}

La naissance de l'expression "L'armée, le peuple et la Résistance » est, comme dans le cas de toutes les expressions de son genre, difficile à cerner puisque son premier usage n'est pas toujours détecté. Cependant, il est probable que le premier emploi soit attribué au Secrétaire Général du Hezbollah², Hassan Nasrallah, au lendemain de la guerre des 33 jours en 2006. Cette guerre, qui oppose l'armée israélienne au Hezbollah, à la suite de l'enlèvement de deux soldats israéliens par ce dernier, réactualise un débat autour de la légitimité de la branche armée du Hezbollah qui a émergé dans les années 80 en pleine guerre civile avec pour objectif la libération des terres libanaises de l'occupation israélienne. La guerre des 33 jours n'oppose pas deux États ou deux armées de ces États ; elle oppose un parti politique doté d'une branche armée et l'armée israélienne. Cette situation remet sur le tapis deux questions importantes: la coexistence d'un groupe armé à l'intérieur de l'État et son statut puissant en raison de son armement lourd par rapport à une armée régulière souffrant de maintes 
insuffisances au niveau de l'armement et de la logistique, sujette aux décisions politiques et à la division communautaire qu'elle doit maintenir en équilibre.

Face à cette instabilité et aux accusations ${ }^{3}$ auxquelles le Hezbollah fait face au lendemain de la fin de la guerre de 2006, cette expression-événement a pour objet de légitimer l'action militaire du Hezbollah et son statut autoproclamé de « Résistance » et de consacrer sa coordination avec l'armée après l'avoir déstabilisée en entrant en guerre contre Tsahal ${ }^{4}$. L'expression arrive à se construire une légitimité dans les discours du Président de la République et la déclaration ministérielle de 2011, avant de se retrouver au centre de la polémique en 2012 avec le discours du Président de la République à l'occasion de la fête de l'armée nationale ${ }^{5}$ puis, en 2013, avec la formation du gouvernement.

3 Cette brève présentation de la situation politique permet de situer le contexte politique qui a permis l'émergence de la formule. L'article se propose tout d'abord de montrer les enjeux politiques qui sous-tendent les faits linguistiques et de préciser en quoi consiste ce que nous avons appelé un "coup de force» linguistique en montrant comment l'usage de la coordination permet d'infléchir le sens des composantes de la formule, contribuant ainsi à la légitimation du Hezbollah. Nous nous appuyons notamment sur les travaux d'A. Krieg-Planque concernant la notion de formule qui nous semblent particulièrement pertinents, notamment à cause de l'accent mis sur les formes de langue. D'autre part, l'article s'intéresse au rôle des médias dans la diffusion (et donc la légitimation) de la formule. Pour ce faire, nous avons sélectionné un minicorpus d'articles de presse de journaux libanais, français et américains parus entre janvier 2010 (probable menace de guerre entre Israël et le Hezbollah) et janvier 2011 (la chute du gouvernement libanais après la démission des ministres du Hezbollah).

4 Ceci dit, nous inscrivons ce travail dans le champ de l'analyse du discours «à la française » (ADF) tout en adoptant une perspective de décloisonnement disciplinaire.

\section{La formule et ses enjeux}

5 En partant des travaux de Faye (1972) et de Fiala et Ebel (1983a, 1983b), Krieg-Planque (2009: 7) propose de concevoir la formule comme un «ensemble de formulations qui, du fait de leur emploi à un moment donné et dans un espace public donné, cristallisent des enjeux politiques et sociaux que ces expressions contribuent dans le même temps à construire ", comme par exemple " purification ethnique » ou encore " développement durable» (Krieg-Planque, 1996, 2010), ou bien avant «État total»(Faye, 1972), «Überfremdung » et "xénophobie» (Ebel \& Fiala, 1983). Ainsi apparait-il que la formule épouse plusieurs formes : elle peut être une unité lexicale simple, complexe, lexico-syntaxique ou encore une séquence autonome.

6 La formule «l'armée, le peuple et la Résistance» est une unité polylexicale dont les mots qui entrent en conjonction forment ce qu'A. Krieg-Planque appelle un "syntagme néologique » en raison du sens lexical nouveau qui se crée par la combinaison insolite de l'armée et du peuple avec la Résistance (voir 2.1, infra).

7 C'est aussi une unité lexico-syntaxique dans le sens où l'alignement des mots se fait dans le cadre d'une opération syntaxique particulière qui est la coordination (ou implicitement via la juxtaposition ${ }^{6}$, voir 2.3., infra). Bien que subissant des changements au fil de sa circulation en discours (voir 4 , infra) qui en assure la naissance et 
l'expansion, elle reste relativement stable. Cette formule stabilise ainsi une représentation via un figement à la frontière langue-discours. Elle se transforme en référent social dans le sens où elle "signifie quelque chose pour tous " (Krieg-Planque, 2010) sans que cela ne signifie qu'elle attire le consensus :

[La notion de référent social] exprime le fait que dans un certain état des rapports de forces sociaux, des formules surgissent dans le langage par rapport auxquelles l'ensemble des forces sociales, l'ensemble des locuteurs sont contraints de prendre position, de les définir, de les combattre ou de les approuver, mais en tout état de cause, de les faire circuler d'une manière ou d'une autre. (Fiala \& Ebel, 1983a : 174).

Ceci nous permet de tirer la dernière caractéristique de la formule : elle est polémique. Et ce caractère polémique est dû aux nombreux enjeux interdépendants qui l'accompagnent: le flou dans la définition des éléments qui la composent; la relation ambivalente entre le Hezbollah et l'armée ; et, surtout, la tentative de légitimation du Hezbollah, de son action et de son identité au sein de l'État libanais, qui permet à ce groupe d'incorporer la Résistance dans la définition du Liban au point d'en faire une marque identitaire.

\subsection{La formule : Un flou linguistique et politique}

9 La formule «L'armée, le peuple et la Résistance » est un instrument de flou dans le sens où les mots qui la composent ont des acceptions vagues et ce, en dépit de son usage dans les discours politiques et médiatiques. En effet, « le caractère dominant d'une formule n'en implique nullement l'homogénéité », nous explique A. Krieg-Planque $(2010: 8)$ car « les mots peuvent changer de sens selon les positions tenues par ceux qui les emploient » (M. Pêcheux, 1971/1990: 140). C'est pour cela qu'elle sera peu employée " sur le mode de la transparence totale " (A. Krieg, $1996: 111)$ : en effet, dans notre corpus, il est rare, presque impossible, de rencontrer, en dehors d'AK, une reprise de la formulation en dehors des guillemets et des renvois aux énonciateurs; c'est une expression qui «ne vas pas de soi » (Authier-Revuz, 1995). La formule est donc le lieu des conflits, des polémiques, des accords et des désaccords, avec, en même temps (et peut-être paradoxalement) un partage du référent social et une connaissance des mots constitutifs qui doivent renvoyer à un sens partagé.

\subsection{1. «L'armée » et « le peuple » : deux composantes de l'État moderne}

10 L'armée: afin de comprendre les enjeux qui sous-tendent cette formule, il faut en premier revenir sur la situation du Liban. Dans le paysage arabe, où les armées ont fomenté des coups d'État afin d'instaurer un pouvoir militaire, le Liban est une exception. L'armée libanaise se constitue au cours du mandat français et se transforme en un symbole d'unité nationale. Le tandem "Armée/Nation » devient un slogan pour certains partis libanais. Or, dans le contexte libanais fortement divisé, et en raison du système confessionnel d'usage dans toutes les institutions, y compris l'armée libanaise, celle-ci n'arrive pas à attirer la sympathie de tous, notamment des musulmans qui l'entrevoient comme une " armée chrétienne » (Moussa, 2012). De plus, l'armée fait face à plusieurs défis : la faiblesse de ses munitions et armement, un éclatement interne favorisé par une répartition confessionnelle et la position ambivalente de l'État à son égard. Ainsi, bien que qualifiée de garante de la cohésion et de l'union nationale, l'armée trouve son rôle et ses fonctions limités par le pouvoir politique. 
11 Le peuple : le mot est cerné d'un flou à la fois linguistique et politique. Si l'on considère qu'il s'agit d'un tout (=la totalité de la population), force est de prendre en considération le fait que le Hezbollah et sa formule ne remportent pas le consensus de l'ensemble du peuple libanais. En même temps, ce même peuple n'est pas un tout homogène, traversé qu'il est par les répartitions confessionnelles et politiques. Quand le Secrétaire Général du Hezbollah lance l'expression, il introduit l'élément " peuple » dans le but de légitimer son action par le parti constitue le fondement de l'État moderne et la source du pouvoir institutionnel ${ }^{7}$. En effet, selon Martin Rojo et van Dijk (1997 : 560-561), la légitimation en tant qu'acte « d'attribution de l'acceptabilité aux acteurs sociaux, actions et relations sociales au sein de l'ordre normatif ${ }^{8}$ se produit dans un contexte d' " actions controversées, d'accusations, de doutes, de critiques ou de conflits sur les relations de groupes, la domination et le leadership ${ }^{9}$. C'est dans un contexte d'accusations au lendemain de la guerre de 2006 que le Hezbollah essaie de légitimer son action qu'il qualifie de résistante à travers la formule et son association à deux éléments constitutifs de l'État libanais.

\subsubsection{Le cas de « la Résistance »}

12 Quant à « la Résistance », il s'agit d'une autoreprésentation et d'une autodénomination du Hezbollah qu'il introduit dans son premier manifeste et fixe sur son drapeau. Elle est ensuite consacrée par la fête officielle de la Résistance (célébrée par un jour férié le 25 mai), par les discours d'investiture des Présidents de la République libanaise tout comme par les déclarations ministérielles libanaises où «Hezbollah » disparaît au profit de " la Résistance ». Mais ce statut est refusé par les pays ayant placé le Hezbollah sur la liste des organisations terroristes.

\section{(1)}

Rendant la politesse à une majorité qui ne cesse de réclamer son désarmement, le Hezbollah a annoncé, après cet incident, que la Résistance se met à la disposition de l'armée. (OLJ, 19.08.2010)

13 L'exemple 1 est archétypal de la relation qui existe entre «la Résistance » et le Hezbollah qui permet au premier terme de devenir une reprise anaphorique naturelle du deuxième dans la presse libanaise. En effet, la relation entre « la Résistance » et le Hezbollah est passée d'une simple affinité à un lien durable et rigide. Ainsi, la Résistance qui référait dans l'histoire du Liban à d'autres groupes comme le Front Libanais ou encore AMAL (afwâju l-muqâwamati l-lubnâniya ${ }^{10}$ ) devient l'apanage du Hezbollah qui émerge comme «la Résistance Islamique ", première dénomination qui montre son idéologie religieuse, avant de passer à «la Résistance [libanaise] » dépouillée de tout caractérisant susceptible de la limiter à une communauté précise.

14 Ce contexte, renforcé par la propagation de la dénomination dans les discours médiatiques et politiques, permet à «la Résistance» dans toutes ses variantes de devenir partie intégrante de l'identité du Hezbollah qui monopolise l'action et la dénomination comme nous le montre le recours à la majuscule et à l'article défini «la qui a comme double objectif de «pointer sur un référent déjà en mémoire 
(fonction de rappel) » et d'« identifier un référent en le singularisant (c'est-à-dire en marquant le caractère d'unicité de l'objet....) » (Benetti, $2008: 165)$.

Mais, en dehors de cette situation caractéristique du Liban, il est peu probable que des lecteurs occidentaux puissent lier «Hezbollah» à «Résistance»: un Français, par exemple, entend, par ce même mot, la Résistance intérieure française qui s'est organisée durant la deuxième guerre mondiale dans le but de libérer la France.

Ainsi, ce lien durable qu'instaure le Hezbollah avec la « Résistance » ne se réalise pas dans les contextes étrangers ce qui pousse la presse française et américaine à recourir à certaines stratégies comme par exemples les notes de la rédaction ou encore certaines reformulations qui permettent à son lectorat d'identifier le référent en question :

(2)

Il y a un dangereux projet qui vise la résistance [le Hezbollah] (LM, 22.07.2010)

Même la presse libanaise se trouve parfois dans l'urgence de rappeler ce lien pour un public non averti :

(3)

$$
\text { 11 حزب الله- و المقصود بذلكالمقاومة }
$$

(AK, 16.10.2010)

(4)

Le leader du PSP s'est adressé aux correspondants de presse, soulignant notamment sa solidarité avec la « résistance » (le Hezbollah). (OLJ, 05.08.2010)

Ce flou qui enveloppe les éléments de la formule participe à la redéfinition de l'élément «État » qui, dans sa définition moderne, ne comprend point de force armée en dehors de son armée régulière. Mais, encore une fois, force est de rappeler l'exception qu'est le Liban qui n'a pas encore pu former cet État moderne. Comme l'explique G. Corm (2012), l'appartenance à une communauté (religieuse, idéologique, politique) l'emporte sur l'appartenance à l'État :

Il y a, en effet, autant de Liban qu'il y a de Libanais, mais aussi autant de Liban qu'il y a de communautés religieuses politiquement agissantes et autant de Liban qu'il y a de visions du monde que produisent les idéologies dominantes dans le monde occidental et le monde arabe. Les Libanais sont d'autant plus sensibles à ces idéologies qu'ils reçoivent chez eux, dès l'enseignement primaire et jusqu'à l'enseignement supérieur, des sensibilités culturelles différentes, imprégnées de ces idéologies et acquises à travers le système fragmenté d'éducation. Les différentes communautés religieuses, de leur côté, ont toujours entretenu des contacts plus ou moins intensifs avec leurs prolongements dans d'autres pays ou avec les pays où se situent des centres spirituels auxquels leurs communautés sont fidèles. 
Cet éclatement a favorisé l'appartenance et l'affiliation à la religion qui régit son système politique et institutionnel libanais, aux diverses idéologies et pays. L'accord de Taëf, conclu au lendemain de la fin des combats de la guerre civile, a sacralisé le communautarisme en aménageant les pouvoirs politiques sur une base de répartition du pouvoir entre communautés religieuses. L'affiliation du peuple libanais à l'État a été affaiblie au profit de la religion, du parti politique, de la région, du clan,... La conception d'un État libanais, souverain et indépendant, laïque et en marge des conflits régionaux, a échoué, ce qui a favorisé la création de micro-états communautaires et régionaux ${ }^{12}$. Cette situation n'a pas été sans influence sur l'acception des mots comme «État », « armée » ou « peuple » et donc sur l'intégration du Hezbollah comme élément constitutif de l'État.

Cette intégration est montrée par "la complémentarité » qui vient marquer une consolidation entre ces quatre éléments, une complémentarité consacrée dans les discours du Secrétaire Général du Hezbollah :

L'armée défend la Résistance et la Résistance a l'honneur d'être défendue par l'armée. La Résistance défend également l'armée, et le peuple défend tant la Résistance que l'armée. C'est la seule équation qui défend le Liban et la seule qui préserve sa dignité. ${ }^{13}$

21 Au niveau pratique, elle est cristallisée par une sorte de coordination entre les deux organismes. Cette collaboration s'est surtout consolidée à la frontière libano-syrienne face aux groupuscules terroristes.

En même temps, bien que le Hezbollah déclare son inscription dans l'État via cette formule tout comme à travers son manifeste de 2009, quand il mène sa guerre contre Israël en 2006, il le fait sans concertation avec l'État. Et quand il décide de prendre part à la guerre en Syrie aux côtés du régime syrien ${ }^{14}$, il le fait indépendamment encore une fois sans revenir à l'État. Ce qui montre une relation ambivalente à l'égard de l'État et de son droit de guerre et de paix que prend en charge l'armée régulière.

\subsection{La relation ambivalente entre l'armée régulière et le Hezbollah}

Cet enjeu remet en cause l'acception de l'armée comme institution de l'État, se rangeant sous l'égide de celui-ci dans les objectifs et les missions. Dans le cas de l'armée libanaise (ou forces armées libanaises), comme nous l'avons déjà expliqué, il s'agit d'une exception dans le paysage arabe et international. Le Hezbollah entretient une relation que l'on pourrait qualifier d'ambivalente avec l'armée régulière : ainsi, la branche armée du Hezbollah ou la Résistance Islamique se présente comme le complément de l'armée libanaise comme le montre l'alignement de quatre éléments (l'armée, la Résistance, le peuple et l'État) dans un même paradigme qui favorise l'idée d'une égalité entre les composantes, voire une complémentarité telle qu'explicitée dans l'exemple suivant :

(5)

M. Joumblatt a mis en relief la formule suivante, à savoir « la complémentarité objective et naturelle entre l'armée, la Résistance, le peuple et l'État ", en sommant certains responsables de cesser de théoriser sur cette question. (OLJ, 06.08.2010) 
part, nous avons l'impression que cette formule remet en cause l'élément « État » qui, dans sa définition traditionnelle, comprend l'armée et le peuple. Mais d'autre part, «la complémentarité » vient marquer une consolidation entre ces quatre éléments. Dans d'autres cas, c'est la Résistance qui arrive à englober l'armée et le peuple qui deviennent deux parties intégrantes de celle-ci, et non l'inverse :

(6)

$$
\text { ومات المتعاقبة، وفي صلب مداولات طاولة الحوار الوطني التي كرّست شرعية سلاح الحزب وشرعت تبحث عن إطار لاستخدامه، وفي صلب }
$$

\subsection{La double légitimation du statut et du rôle du Hezbollah au sein de l'État libanais}

Les deux premiers enjeux exposés concourent à élucider le troisième, le plus important aussi: la formule cristallise la légitimation du statut du Hezbollah comme «la Résistance » ainsi que son appropriation du droit de guerre et de paix au sein de l'État libanais.

Dans la formule, « Résistance » reprend donc « Hezbollah » (voir supra) pour l'associer à deux composantes traditionnelles des États - le peuple et l'armée. Pour rappel, il s'agit ici de l'État au sens occidental du terme : une structure moderne ayant émergé après l'appropriation du droit de guerre et de paix par l'État. Selon Grotius (1729), ce droit se concentre en l'État qui peut déclarer des guerres justes dont le motif est de s'autodéfendre et des guerres coercitives pour punir ceux qui violent la loi. Dans les deux cas, le motif doit être sérieux et une guerre ne peut subvenir qu'une fois les modes de règlements pacifiques épuisés. Concentrer le droit de la guerre et de la paix dans l'État, source, pivot et monopole des pouvoirs, c'est le confier à l'armée du pays. Or, au Liban, ce rôle traditionnel de l'armée se trouve aussi assuré par le Hezbollah qui, à travers le lancement de la formule, compte légitimer son statut et son rôle. Ainsi, agréer la formule, c'est accepter le statut et le rôle que le Hezbollah souhaite jouer au sein de l'État, alors que la refuser, c'est réfuter ce même statut et ce même rôle et suggérer que le Hezbollah est une milice en marge de l'État libanais ${ }^{16}$.

L'étude syntaxique des éléments qui forment la formule dévoile l'existence d'une jonction implicite (juxtaposition) ou explicite (coordination) entre les trois composantes, dont deux - " peuple » et " armée » - portent le trait [+légitime]. Comme le montrent Guilhaumou et al. (1994) dans leur travail sur « Du pain et... » : la coordination se fait en général au niveau d'éléments de même nature; quand des éléments différents s'assemblent, un supplément de sens est produit. Dans le travail de Guilhaumou et al., le vieux thème de la demande de moyens de subsistance devient un thème politique une fois uni, via la coordination, à " liberté » : ainsi, «liberté » a en quelque sorte « politisé » le pain. Ce « travail de la grammaire produit directement des effets discursifs $» .^{17}$

Dans le cas de notre formule, la coordination assure l'association et le classement des éléments qui composent la formule dans une relation holonyme/méronyme ${ }^{18}$ où les

TIPA. Travaux interdisciplinaires sur la parole et le langage, 33 | 2017 
trois éléments se comportent comme les constituants méronymiques de l'holonyme « Nation ou État » : le Liban est composé des trois éléments que comprend la formule. Or, si « armée » et «peuple » font partie de l'acception classique de l'État, « Résistance » est un coup de force linguistique qui a pour but de légitimer une entité qui n'en fait pas partie à travers son association aux deux premières.

Cette hypothèse montre que si " l'analyse linguistique construit le paradigme des termes ", « l'étude discursive met en lumière les affrontements qui se jouent autour des coordinations. Au-delà de la question grammaticale de la bonne formation de la coordination, on se situe alors au point où se débat la légitimité, l'acceptabilité de la coordination $» .{ }^{19}$

\section{Une formule polémique}

Les enjeux susmentionnés, qui sous-tendent la formule et que prennent en charge d'expliciter les divers outils linguistiques, concourent à lui octroyer son caractère polémique. D'ailleurs, la formule, étant elle-même un enjeu, est par essence polémique. Sa circulation massive dans les médias et son appropriation par plusieurs locuteurs et dans divers contextes augmentent son degré de polémique. En effet, «la formule circule à l'appui de certains usages qui lui donnent un caractère conflictuel ou problématique, et à l'appui d'événements ou d'autres discours qui motivent son utilisation, donnent des raisons aux locuteurs de recourir à elle d'une manière ou d'une autre » (Krieg-Planque, 2009 : 43). La formule devient très rapidement un lieu de débat et de confrontation, une " arène » entre les pro-Résistance et les anti-Résistance qui travaillent à la «dynamiter " pour essayer d'ôter au Hezbollah la légitimité qu'il a essayé d'instaurer en élevant sa Résistance au même rang que le peuple et l'armée.

L'usage même de la formule devient problématique puisqu'il stigmatise son énonciateur en signalant son adhésion à la politique du Hezbollah. D'où la nécessaire distanciation à travers des opérations d'opacification comme l'autonymie qui, tout en offrant au journaliste l'occasion de respecter les paroles d'autrui, lui permet aussi de gloser sur l'inadéquation de la formule avec le contexte auquel elle renvoie :

(7)

A slogan often repeated by Lebanese officials, "The people, the army, the resistance will protect Lebanon," shows the tacit support the government still provides to Hezbollah to resist Israel even though Israeli troops withdrew from south Lebanon in 2000. ${ }^{20}$ (WP, 13.08.2010)

(8)

$$
\begin{aligned}
& \text { بدعة “ الجيش والشعب والمقاومة” آخرها التسوية التي افضت الى اختراع } \\
& \text { 21 21التي لم يعهدها بلد غير لبنان . لمان والمع }
\end{aligned}
$$$$
\text { (AN, 30.09.2010) }
$$

La glose métalinguistique se traduit par des mots génériques "neutres" comme "slogan ", « équation ", «formule ", « trinôme », etc. ou par des mots plus marqués 
comme «hérésie " chez Ali Hamadé dans $\mathrm{AN}^{22}$. Ali Hamadé, auteur de l'article en question, appartient au camp opposé au Hezbollah et souligne par l'emploi du mot « hérésie » non seulement le caractère inacceptable de la formule, mais aussi et surtout l'erreur « doctrinaire » que cette formule fait passer comme vérité consensuelle.

Même si la formule s'impose dans le discours de la presse libanaise (OLJ, AN et AK), elle n'arrive pas à s'installer dans un cadre consensuel. En effet, si AK se l'approprie, l'OLJ et AN la manient avec des pincettes en raison de la stigmatisation que son usage pourrait provoquer.

Peu fréquente, presque inexistante dans la presse française et américaine (aucune occurrence n'est relevée dans le NYT), cette formule reste confinée aux frontières du discours rapporté.

\section{Substituabilité, productivité et changement de la formule}

À travers les usages en discours, la formule connaît des changements. Son caractère relativement figé, évoqué plus haut, constitue une de ses propriétés, il est vrai, sans pour autant l'aliéner. Ainsi, une formule est-elle sujette aux additions ou soustractions ou aux changements qu'imposent les traductions ${ }^{23}$, voire l'inversement de l'ordre des éléments qui la composent :

(9)

Hassan Nasrallah a insisté sur l'interdépendance de l'armée et de la résistance, affirmant que cette nouvelle équation "défensive » venait de connaître son «baptême du sang » (LF, 05.08.2010)

De tels remodelages relèvent de la " productivité lexicologique » de la formule (KriegPlanque, 2009) qu'il faut saisir au-delà des discours et des locuteurs, à travers des faits de reprise, de reformulation, de régularité, de circulation, de dispersion et d'écho.

La formule subit surtout des expansions et des réductions. Les éléments substituables dans les deux cas seront " le peuple » ou, plus rarement, "l'armée » alors que " la Résistance » formera le noyau dur de la formule. Parfois, d'autres éléments comme " gouvernement " ou "État " seront parfois privilégiés dans le but de doter la « Résistance » de la légitimité demandée :

(10)

L'armée libanaise applique le slogan figurant dans la déclaration de politique générale du gouvernement, qui dit que le gouvernement, l'armée, le peuple et la Résistance défendront ensemble la patrie, relève M. Azzi. (OLJ, 06.08.2010) 
(11)

M. Joumblatt a mis en relief la formule suivante, à savoir « la complémentarité objective et naturelle entre l'armée, la Résistance, le peuple et l'État ", en sommant certains responsables de cesser de théoriser sur cette question. (OLJ, 06.08.2010)

(12)

Selon lui, ces liens de plus en plus étroits renforcent la stabilité dans la région, ajoutant qu'il se sent très heureux lorsqu'il voit " cette osmose entre le peuple, la Résistance et le gouvernement au Liban ", assurant aussi que c'est le meilleur moyen de couper la voie aux complots ourdis par les ennemis du Liban (OLJ, 6.08.2010)

La réduction, qui permet de recentrer la formule autour de deux pôles, avec «la Résistance " comme noyau dur, favorise, selon A. Krieg-Planque (2009 : 74-75) une certaine et « relative évaporation du sens du segment disparu, évaporation qui aboutit à un certain flottement sémantique dans lequel s'engouffrent des polémiques »ou, comme le montre l'exemple suivant, « l'utilisation de la séquence ainsi réduite pour la désignation d'autres objets, emploi (...) qualifié de « propagation » de la formule... » :

(13)

Non seulement l'armée libanaise a réagi avec beaucoup de courage, alors qu'elle n'a pratiquement pas les moyens techniques d'affronter les soldats israéliens, mais elle a aussi clairement montré que nul ne peut miser sur une modification de l'équation arméeRésistance et de la coopération entre elles (OLJ, 10.09.2010).

\section{Conclusion}

Nous avons pu travailler à partir d'une reprise ${ }^{24}$ cristallisant une représentation du Hezbollah - «Résistance » - la formule "L'armée, le peuple et la Résistance » qui circule dans le cadre d'un discours rapporté ou s'enchaîne dans le cadre d'un consensus ou d'un conflit. Cette formule nous a paru indispensable à traiter car elle met en question divers enjeux. Elle a surtout ouvert la réflexion sur un questionnement à propos de la conception de l'État en montrant le fossé qui existe entre une conception occidentale d'un État unifié, basé sur une centration du pouvoir armé par et dans l'État à travers l'armée régulière, et une conception libanaise d'un État éclaté en communautés où le pouvoir armé a été pris par des groupuscules divers, y compris le Hezbollah. Cette formule cristallise aussi l'enjeu lié à la légitimité que gagne le Hezbollah non seulement en s'auto-désignant comme "Résistance", mais aussi en s'amalgamant à « armée » et à " peuple " grâce à un effet syntaxique (la coordination). Ce que nous pouvons dégager de l'étude du mini-corpus qui s'articule autour de cette formule c'est que, bien que le Hezbollah ait réussi à faire circuler son auto-désignation 
et à imposer sa formule, il n'a pas pour autant garanti une adhésion à la représentation positive qu'elle véhicule, même au niveau des journaux libanais.

\section{BIBLIOGRAPHIE}

Amossy, R., Krieg-Planque, A. \& P. Paissa (2014) La formule en discours. Perspectives argumentatives et culturelles, Repères-Dorif, 5 .

Asmar, P. (2013) Une analyse discursive du nom et des représentations du Hezbollah dans la presse libanaise, française et américaine (2010-2011), Thèse de doctorat, Université Sorbonne Nouvelle-Paris 3 et Université Libanaise, $416 \mathrm{p}$.

Authier-Revuz, J. (1995) Ces mots qui ne vont pas de soi. Boucles réflexives et non-coïncidences du dire, Paris: Larousse, $790 \mathrm{p}$.

Benetti, L. (2008) L'article zéro en français contemporain : Aspects syntaxiques et sémantiques, Berne: Peter Lang, $187 \mathrm{p}$.

Corm, G. (1992) Inquiétudes libanaises, Le Monde Diplomatique, janvier 1992, p. 32.

Corm, G. (2012) Réussir la réforme politique au Liban, Tumultes, 38-39, p. 329-340.

Fiala, P. \& M. Ebel, (1983a) Langages xénophobes et consensus national en Suisse (1960-1980) : discours institutionnels ; la médiatisation des conflits, Neuchâtel: Université de Neuchâtel, 432-XXII p.

Fiala, P. \& M. Ebel, (1983b) Sous le consensus, la xénophobie. Paroles, arguments, contextes (1969-1981), Lausanne: Institut de science politique, 432-XXII p.

Faye, J.-P. (1972) Langages totalitaires. Critique de la raison de l'économie narrative, Paris: Hermann, $771 \mathrm{p}$.

Grotius, H. (1729) Le droit de la guerre et de la paix, vol. 2, trad. de J. Barbeyrac, édition nouvelle, 587 p.

Guilhaumou, J., Maldidier, D. \& R. Robin (1994) Discours et archive: expérimentations en analyse du discours, Bruxelles: Mardaga, 218 p.

Haroche, C., Henry, P. \& M. Pêcheux (1971) La sémantique et la coupure saussurienne : langue, langage, discours, Langages, 24, p. 93-106.

Krieg, A. (1996) La « purification ethnique » dans la presse. Avènement et propagation d'une formule, Mots, 47, p. 109-126.

Krieg-Planque, A. (2007) Travailler les discours dans la pluridisciplinarité. Exemples d'une 'manière de faire' en analyse du discours, in Bonnafous, S. \& M. Temmar (dirs), Analyse du discours et sciences humaines et sociales, Paris: Ophrys, p. 57-71.

Krieg-Planque, A. (2009) La notion de formule en analyse du discours, Franche-Comté: Presses Universitaires de Franche-Comté, 148 p.

Krieg-Planque, A. (2010) La formule « développement durable » : un opérateur de neutralisation de la conflictualité, Langage et Société, 134, p. 5-29. 
Mortureux, M.-F. (2008) La Lexicologie entre langue et discours, Paris: Armand Colin, 212 p.

Moussa, N. (2012) L'armée libanaise : une exception dans le paysage militaire arabe ?, Les Champs de Mars, 23, La place et le rôle des armées dans le monde contemporain.

Martin Rojo, L. \& T. A. van Dijk (1997) “There was a problem and it was solved!” Legitimating the expulsion of "illegal" immigrants in Spanish parliamentary discourse, Discourse and Society, 8, 4, p. 523-567.

\section{NOTES}

1. Il s'agit d'un mini-corpus constitué à partir d'un gros corpus de travail collecté pour notre thèse de doctorat.

2. Le Hezbollah a émergé dans les années 80 au Liban comme branche armée ayant pour but la libération du Sud du Liban, avant de s'organiser en parti politique doté d'une branche armée.

3. C'est le Hezbollah qui entre en guerre et non l'État et ce, sans concertation avec l'armée régulière. De plus, le bilan de la guerre de 33 jours est catastrophique au niveau humain et matériel.

4. L'armée de défense d'Israël. En juillet 2006, à la suite d'un accrochage entre le Hezbollah et les forces israéliennes à la frontière, le premier capture deux soldats israéliens dans le but de négocier un échange avec des prisonniers libanais en Israël. Après avoir échoué à sauver les deux soldats, Israël entre en guerre contre le Hezbollah. La guerre de 2006 a duré 33 jours.

5. Au lendemain de la fête de l'armée libanaise, paraît dans le numéro du 2 août 2012 de l'OLJ un article intitulé «Sleiman dynamite le triptyque armée-peuple-résistance »: "L'allocution du président Sleiman - très attendue cette année - a été axée sur un sujet de brûlante actualité, à savoir le problème des armes illégales et de la place que devrait occuper l'armée dans toute stratégie de défense. Il a affirmé sur ce plan que «la souveraineté, la sécurité et l'usage de la force sont du seul ressort de l'armée », l'usage de la force étant «un droit exclusif dont jouit l'État ».

Disponible en ligne: www.lorientlejour.com/category/\%C3 \%80+La+Une/article/771456/ Sleiman+dynamite_le_triptyque_armee-peuple-resistance.html. [Consulté le : 02.08.2012]

6. En effet, nous avons repéré deux variantes de la formule: une coordonnée («L'armée, le peuple et la Résistance ») et l'autre juxtaposée ("L'armée, le peuple, la Résistance »). Cette variabilité est due à un usage qui défige la formule comme nous le verrons dans la quatrième partie.

7. «Son principe [de la République française] est : gouvernement du peuple par le peuple et pour le peuple. La souveraineté nationale appartient au peuple qui l'exerce par ses représentants et par la voie du référendum. Aucune section du peuple ni aucun individu ne peut s'en attribuer l'exercice ». Constitution de 1958, art. 2 et 3.

8. Citation originale: "Attributing acceptibility to social actors, actions and social relations within the normative order".

9. Citation originale : "controversial actions, accusations, doubts, critique or conflict over groups relations, domination and leadership".

10. Détachement libanais de résistance, fondé par l'Imam Moussa Sadr en 1975 et connu comme le Mouvement des dépossédés (harakat al-mahroumin).

11. Ma traduction : La résistance - le Hezbollah

12. La sécurité personnelle que promeuvent certaines régions, demandant une indépendance de l'État libanais, devient de plus en plus en vogue. Les règlementations des tribus affirment leur suprématie au détriment des réglementations étatiques.

13. Discours du Secrétaire Général du Hezbollah le 3 août 2010. 
14. Le Hezbollah est entré en guerre aux côtés du régime syrien en 2011 avant de l'admettre officiellement à travers un discours de son Secrétaire Général en 2013. Selon ce discours, l'intervention du Hezbollah en Syrie serait justifiée par deux raisons : la protection du tombeau de Zeinab, lieu saint chiite, et des villages frontaliers libanais.

15. Ma traduction : En réalité, le parti a réussi à introduire son choix de résistance au cœur de la stratégie de défense de l'état libanais, en l'évoquant au cœur des déclarations ministérielles des gouvernements successifs, et de la table de dialogue national qui ont consacré la légitimité de l'armement du parti et ont commencé à rechercher un cadre pour son usage, et au cœur d'une équation ayant fait de l'armée et du peuple une partie intégrante de la Résistance, et non l'inverse.

16. Au lendemain de la fin de la guerre civile, l'accord de Taëf a stipulé que tous les groupuscules libanais et non libanais devraient se désarmer. Or, en plus des factions palestiniennes, le Hezbollah est le seul groupe libanais à ne pas avoir remis son armement à l'état.

17. Guilhaumou et al. (1994: 146).

18. Selon Mortureux (2008 [1997]), l'holonymie/méronymie est une relation sémantique entre des mots dont l'un (holonyme) dénomme un ensemble dont les autres (méronymes) dénomment les parties.

19. Guilhaumou et al., op.cit., p. 99.

20. Ma traduction: Un slogan souvent répété par les responsables libanais, «Le peuple, l'armée, la résistance vont protéger le Liban ", montre le soutien tacite que le gouvernement assure au Hezbollah pour qu'il résiste à Israël, en dépit du retrait des troupes israéliennes du Sud du Liban en 2000.

21. Ma traduction : Le dernier compromis a abouti à l'invention de l'hérésie «L'armée, le peuple et la résistance » qu'aucun pays, autre que le Liban, n'a connu.

22. Un journal libanais en langue arabe plutôt hostile au Hezbollah.

23. La formule que nous étudions a d'ailleurs émergé en arabe avant d'être traduite en français et en anglais.

24. La formule fait partie d'un travail plus développé sur les représentations du Hezbollah dans la presse libanaise, française et américaine. À ce sujet, voir Asmar (2013).

\section{RÉSUMÉS}

$\mathrm{Au}$ lendemain de la guerre des 33 jours émerge une expression-événement dans le discours du Secrétaire Général du Hezbollah. "L'armée, le peuple et la Résistance » (al-jaych, al-cha'b wa almouqâwama) n'a pas seulement pour but de consacrer le Hezbollah comme étant la Résistance, mais aussi cette formule se double d'enjeux politiques importants qui sous-tendent les faits linguistiques.

Dans ce contexte, notre article s'intéressera en premier aux enjeux que cette formule cristallise et qu'explicitent les formes de langue ; et, en deuxième, au rôle des médias dans la diffusion (et donc la légitimation) de la formule.

Dans un premier temps, nous présenterons le contexte politique qui a favorisé l'apparition de cette expression avant de revenir sur son adéquation avec les critères de la formule. Ensuite, nous tirerons ses enjeux politiques à travers une analyse linguistique dans un mini-corpus ${ }^{1}$ d'articles de presse libanaise, française et américaine, en français, arabe et anglais, appartenant à plusieurs lignes éditoriales et affiliations politiques. 
A formula is "a set of formulations which, because of their use at a given moment and in a given public space, crystallize political and social issues that these expressions contribute at the same time to building" (Krieg-Planque, 2009). "The army, the people and the Resistance" (al-jaych, alcha'b wa al-mouqāwama) is a formula that follows the criteria researchers (Faye; Fiala \& Ebel; Krieg-Planque) have set to define for this kind of expressions: it shows a crystallized character, it enrolls in a discursive dimension, it functions as a social referent, and it displays a polemical aspect.

The article aims at extracting the political issues underlying the linguistic facts and specifying what we have called a linguistic "coup de force" by showing how the use of coordination makes it possible to change the meaning of the components of the formula, thus contributing to the legitimization of Hezbollah. The article also focuses on the role of the media in circulating the formula.

The emergence of this formula, as in the case of all expressions of its kind, is difficult to identify since its first use is not always traceable. However, the first use is most probably attributed to Hezbollah's Secretary General Hassan Nasrallah in the aftermath of the 33-day war in 2006. This war opposed Hezbollah to Israeli forces and led Hezbollah to face instability and accusations.

The purpose of this expression is to legitimize Hezbollah's military action and its self-proclaimed status of "Resistance" and to reinforce its coordination with the army after having destabilized it.

However, this formula is associated to a number of political issues which are concretized by forms of language:

1) The political and linguistic vagueness of the formula, which is expressed through the impreciseness of the three words that compose the formula: "the army", "the people" and "the Resistance."

2) The ambivalent relationship between the Lebanese army and Hezbollah which is synthetized through corpus examples, showing on one hand the enrolment of Hezbollah and its Resistance within the framework of the Lebanese State, and on the other hand its independence of action and decision.

3) The last, but most important issue is the legitimization of the status and role of Hezbollah within the Lebanese state. Through explicit coordination (or implicit juxtaposition) between the three elements, of which two are considered as legitimate elements of the State ("the army" and "the people"), Hezbollah aims to legitimize its action and status as the Resistance. This legitimization "[attributes] acceptability to social actors, actions and social relations within the normative order" within a framework of "controversial actions, accusations, doubts, critique or conflict over groups' relations, domination and leadership" (Martin Rojo \& van Dijk, 1997: 560-561).

After analyzing the three main issues that the formula carries, we tackle its polemic aspect which makes it quite impossible to appropriate it without risking a stigmatization, justifying the use of the press of forms of reported speech while glossing over its linguistic adequacy, before moving to examine the changes the formula undergoes in media discourse.

INDEX

Mots-clés : formule, Hezbollah, polémique, discours, presse écrite, consensus et conflit

Keywords : formula, Hezbollah, controversy, discourse, written press, consensus and conflict 
AUTEUR

\section{PASCALE ASMAR}

Université Sorbonne Nouvelle - Paris 3

Université Libanaise

pascale.asmar@hotmail.com

pascale.asmar@ul.edu.lb 